\title{
Reviewer Acknowledgements for Global Journal of Health Science, Vol. 9, No. 2
}

Global Journal of Health Science wishes to acknowledge the following individuals for their assistance with peer review of manuscripts for this issue. Their help and contributions in maintaining the quality of the journal are greatly appreciated.

Global Journal of Health Science is recruiting reviewers for the journal. If you are interested in becoming a reviewer, we welcome you to join us. Please find the application form and details at http://recruitment.ccsenet.org and e-mail the completed application form to gjhs@ccsenet.org.

\section{Reviewers for Volume 9, Number 2}

A. B. Ruzilawati

A. N. B. Johnston

Abdel-Hady El-Gilany

Achmad Nizar Hidayanto

Alexandre F. Marques

Ali Farhoudian

Amir Elalouf

Ana Cristina Rodríguez-Dehli

Angel M. Foster

Aniel Jessica Leticia Brambila-Tapia

Bonny J. Taylor

Carlos Santibanez

Chithananda Mudalagiriyappa

Christos Andreou

Christos Argyropoulos

Chunhua Ma

Dandan Huang

Daniela-Tatiana Agheorghiesei

Deepu David

Donna J. Sauls

Dorota Szcześniak

Dumilah Ayuningtyas

Emmanuel Kamal Saba

Erica Leeanne Lizano

Erik J. Koornneef

Erik R.M. Eckhardt

Fabian Sanchis-Gomar
Fatih Büyükcam

Fayaz Mohammed Khazi

Feitong Wu

Friedrich M. Von Recklinghausen

Guy Wachtel

Hamid Kassiri

Helen Benedict Lasimbang

Hidetaka Tamune

Ibrahim Elmadbouh

James Yun-Bae Kim

Jaqueline K. Eserian

Jau-Shya Lee

Ji Yonghua

Jovana Cvetković

Juan M. P. Nañagas

Júlia Matzenbacher Dos Santos

Juliana Martins Pinto

Kara L. Holloway

Karin Hummel

Laura A. Zinsser

Leonard O'sullivan

Marlene Toufic Chakhtoura

Masaaki Minami

Maxwell Osei-Ampofo

Metin Ersoy

Mohammad Ali Morowati Sharifabad

Mohammad Ghannaee Arani
Nagat S. El-Shmaa

Natalie Callis

Nilgun Ulutasdemir

O. A. Oyelami

Oznur Sen

Pedro Gallo

Reza Hakkak

Rong-Sen Yang

Rose Omari

Rouhullah Dehghani

Rui Torres

Russell D. Macdonald

S. Beukes

Salim Chahin

Salisu Abubakar

Samantha J. Brooks

Samira Schultz Mansur

Sanjeev Aneja

Sarah B. Maness

Saranrat Wittayanukorn

Shunmugaperumal Tamilvanan

Sofia Zwedberg

Stanislaw Szlufik

Taofiki A. Sunmonu

Yadollah Abolfathi Momtaz

Zohra Aydi 